\title{
Advances in Electromagnetic Modelling through High Performance Computing
}

\author{
K. Ko, N. Folwell, L. Ge, A. Guetz, L. Lee, Z. Li, C. Ng, E. Prudencio, G. Schussman, \\ R.Uplenchwar, L. Xiao \\ Stanford Linear Accelerator Center, 2575 Sand Hill Road, Menlo Park, Calif. 94025 \\ USA
}

Kwok@slac.stanford.edu

\begin{abstract}
Under the DOE SciDAC project on Accelerator Science and Technology, a suite of electromagnetic codes has been under development at SLAC that are based on unstructured grids for higher accuracy, and use parallel processing to enable large-scale simulation. The new modelling capability is supported by SciDAC collaborations on meshing, solvers, refinement, optimization and visualization. These advances in computational science are described and the application of the parallel eigensolver Omega3P to the cavity design for the International Linear Collider is discussed.
\end{abstract}

\section{Introduction}

SciDAC (Scientific Discovery through Advanced Computing) [1] is a Department of Energy (DOE) initiative to advance high performance computing and promote its use in simulations devoted to the applications in its Office of Science (SC) program offices. With support from the High Energy Physics's (HEP) SciDAC Accelerator Science and Technology (AST) project, SLAC is developing a new electromagnetic modelling capability to enable the large-scale simulations needed for improving existing accelerators and for optimizing the designs of proposed facilities. Particle accelerators are essential tools for scientific discovery across SC and as such, they are among the largest and most complex scientific instruments in the world. Given their significant cost, it deems necessary that the most advanced numerical tools and resources be brought to bear on these machines to ensure and maximize DOE's returns of investment on Science.

Electromagnetic software for modelling accelerating cavities have been in existence for decades, first in 2D and more recently in 3D, with standard codes like MAFIA, Microwave Studio and HFSS now in routine use by the community. The first two codes are based on the finite difference (FD) method while the last employs the finite element (FE) method. In the FE method, the unstructured mesh models curved surfaces better and the higher order basis functions can increase accuracy substantially. Presently, these codes are memory limited as they only run on a single computer while the latest supercomputers consist of thousands of processors with a significantly larger total memory.

Under SciDAC, SLAC is developing parallel finite element electromagnetic codes [2] to obtain gains in accuracy, problem size and solution speed by harnessing the compute power and exploiting the huge memory of the latest SC's supercomputers, e.g. the IBM/SP (Seaborg) at NERSC and the 
Cray/X1E (Phoenix) at NLCF. The code suite to date includes the eigensolver Omega3P, the S-matrix solver S3P, the time-domain solver T3P, and the particle tracking code Track3P. This paper reports on the advances made in Omega3P and the code's application to the design of a new Low-Loss (LL) accelerating cavity [3] for the International Linear Collider (ILC) [4].

\section{Low-Loss Cavity Design for the ILC}

An international team comprising DESY, KEK, JLab, FNAL and SLAC is collaborating to realize an alternate cavity design for the ILC, the Low-Loss (LL) design. The LL cell shape has $23 \%$ less cryogenic loss plus higher accelerating gradient than the standard TESLA shape. Recent experiments reported [5] that a single LL cell can reach $46.5 \mathrm{MV} / \mathrm{m}$ as compared to the $35 \mathrm{MV} / \mathrm{m}$ achieved with TESLA cells [6]. The next step now is to demonstrate this high gradient in a 9-cell cavity as required for the ILC. In operation, beam generated Higher-Order-Modes (HOMs) in the cavity constitute the wakefields that can dilute the beam emittance and if uncontrolled, disrupt the transport of bunch trains down the linac. Achieving high gradient for increased acceleration and providing HOM damping that ensures beam stability are the two critical issues facing the ILC cavity design. Based on the TESLA design, the LL cavity consists of 7 interior cells with 2 end cells connected to one fundamental power coupler (FPC) and two Higher-Order-Mode couplers (HMCs) as shown in Fig. 1. The TESLA design has been in existence since the early 90's although no direct simulation of the entire cavity had been done until now because of two obstacles. One was the significant computing resources required to model the disparate length scales of the cavity accurately, from the cell shape to the fine features in the couplers. Another was the lack of a complex eigensolver that can directly calculate the external Q (Qext) of a mode as a result of power flow out of the cavity through the couplers. Advances made in Omega3P under SciDAC have overcome both difficulties
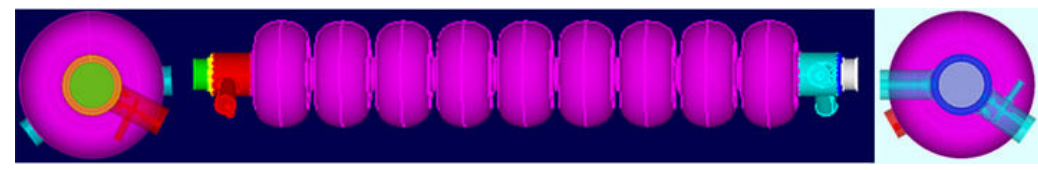

Figure 1 Model of the ILC LL cavity design - side-view (middle) showing the 9 cells, and the endviews showing front HOM coupler (left) and the rear FPC/HOM couplers (right) respectively.

\section{Parallel Finite Element Eigensolver Omega3P}

\subsection{Combined capability of FE method and parallel processing}

Omega3P is formulated on tetrahedral mesh with curved surface and employs up to $6^{\text {th }}$ order FE basis functions for field representation. The code runs on distributed memory computers through MPI. To illustrate the combined capability of FE method and parallel processing, consider the simplified model shown below. The fundamental mode frequency was calculated by Omega3P with $\mathrm{p}=1$ and $\mathrm{p}=2$ elements for increasingly dense meshes and the results are shown in Figure 2 where the frequency error is plotted against required memory for solution. It shows that for the same memory, quadratic elements provides much high accuracy and that to model an entire cavity of 9 cells with reasonable accuracy, memory available on multi-processors are required.
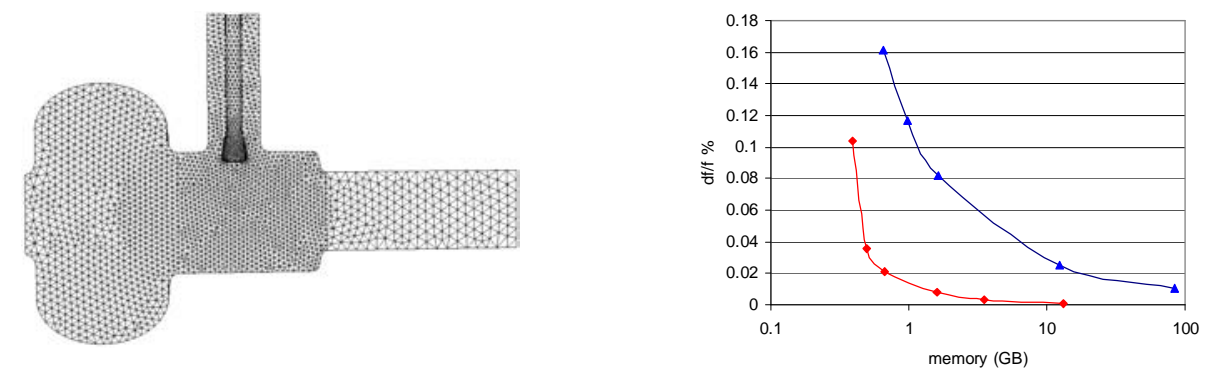

Figure 2. Mesh of simplified test cavity and convergence of frequency error with required memory (blue is linear elements, red is quadratic elements). 


\subsection{Complex Eigensolver for RF Cavities Coupled to Matched Waveguides}

The FPC and HMCs in the LL cavity enable power flow into and out of the cavity through matched coaxial waveguides. As a result, the eigenfrequency of a normal mode in this waveguide loaded cavity becomes complex. The external coupling is measured by the external quality factor Qext which is one half the ratio of the real to the imaginary part of the eigenfrequency. The complex eigenfrequency is obtained by solving the nonlinear eigenvalue problem:

$$
\mathrm{K} x+i \sum_{k} \sqrt{k^{2}-k_{c}^{2}} \mathrm{~W}(k) x=\mathrm{M} x
$$

Where

$$
\mathrm{K}_{i j}=\int_{\Omega}\left(\nabla \times \mathrm{N}_{i}\right) \bullet \frac{1}{\varepsilon}\left(\nabla \times \mathrm{N}_{j}\right) d \Omega, \mathrm{M}_{i j}=\int_{\Omega} \mathrm{N}_{i} \bullet \mu \mathrm{N}_{j} d \Omega \text {, and } \mathrm{W}(k)_{i j}=\int_{\Gamma_{k}}\left(\mathrm{n} \times \mathrm{N}_{i}\right) \bullet\left(\mathrm{n} \times \mathrm{N}_{j}\right) d \Gamma
$$

with $\mathbf{N}$ being the Nedelec edge elements, $k_{c}$ is the waveguide cutoff and $k$ is the $\omega / c$. With the waveguide ports closed (no waveguide terms involving $\mathbf{W}$ ), Eq. (1) reduces to the standard eigenvalue problem for a lossless cavity. If there is only a single waveguide cutoff, Eq. 1 can be transformed to a quadratic eigenvalue problem $(\mathrm{QEP})$ :

$$
\lambda^{2} \mathrm{M} x-i \lambda \mathrm{W} x+\left(k_{c}^{2} \mathrm{M}-\mathrm{K}\right) x=0
$$

with $\lambda=\sqrt{k^{2}-k_{c}^{2}}$.

In collaboration with UC Davis and LBNL, two algorithms have been developed to solve the QEP. The first algorithm follows the Second Order Arnoldi (SOAR) method [7] which generates an orthogonal basis set for the second order Krylov subspace. When used with the standard Rayleigh-Ritz orthogonal projection technique, SOAR can be directly applied while preserving its structure and properties. The second algorithm is a straightforward scheme called Self-Consistent Iteration (SCI) [8] which is also applicable to the general nonlinear eigenvalue problems with multiple waveguide modes. It uses simple successive approximation starting with the linear solutions without the waveguide terms and iterates till the residual of the nonlinear eigensystem is below a specified tolerance. While lacking a theory to guarantee convergence, the method often converges in 3 to 5 iterations in practice.

\section{Omega3P Calculations of HOM Damping in the LL Cavity}

Omega3P with quadratic elements was used to model the first designs of the ILC low-loss cavity. Figure 3 shows the mesh model of the cavity partitioned with ParMETIS onto different processors. Of particular importance in the simulation is the damping that can be achieved by the given set of HOM couplers. With the complex eigensolver, Omega3P calculated the complex eigenfequencies of the modes in the lowest five dipole bands from which the Qext, the figure of merit for damping, can be obtained and the results are shown Figure 4. When multiplied by their respective R/Q, it was found that a well trapped mode in the third band could pose a problem to beam stability because of its high shunt impedance. Efforts are ongoing to optimize the cavity to reduce the Qext of this dangerous mode. On the IBM SP at NERSC, these computations required 300 GB of memory on 738 CPUs and generated at the rate of 18 modes per hour.

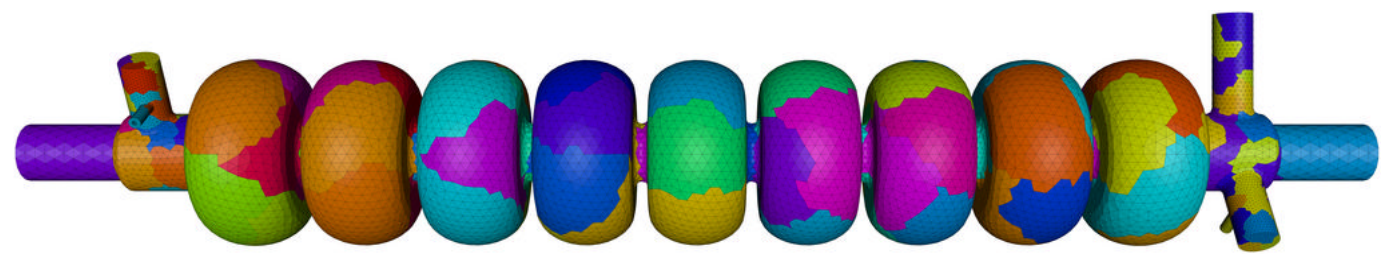

Figure 3. Partitioned mesh model of the Low-Loss cavity design for the International Linear Collider. 


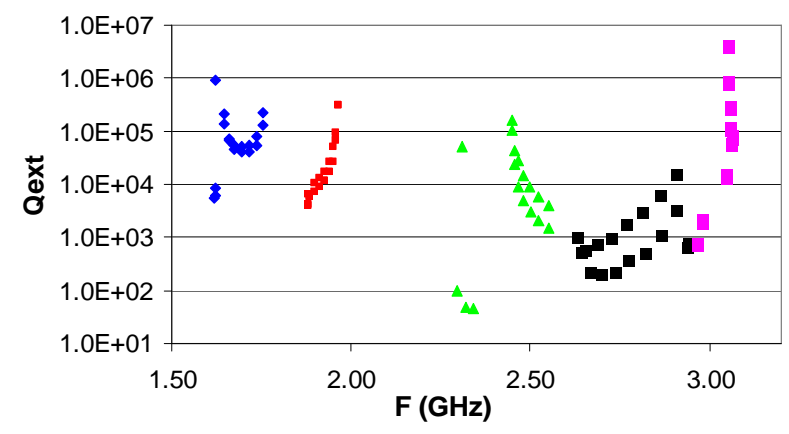

Figure.4. Omega3P results for the external Qs of the dipole modes in the LL cavity.

\section{Advances in Computational Science for Omega3P}

Parallel electromagnetic modeling is still in the early stage of development so therefore much work remains to be done in many areas of computational science. In addition to the effort on the parallel nonlinear eigensolver described above, SLAC is collaborating with its SciDAC partners on:

\subsection{Visualization}

In collaboration with UC Davis, new visualization tools [9] are being developed to help analyze field patterns of HOM modes in the ILC cavity so that their effect on the beam can be better understood. A new mode rotation effect was uncovered when visualizing the time evolution of the fields obtained from the Omega3P complex eigenvectors. This occurs in a fully 3D geometry with no symmetry when the degeneracy of a dipole mode is split and the two degenerate modes couple to each other. The coupling arises when the damping due to the HOM couplers introduces a resonance width that exceeds the frequency separation of the two modes. As a result, the mode mixing leads to field patterns that are elliptically polarized and they rotate over a wave period. A snapshot of the two rotating modes is shown in Figure 5. Work is underway to study the consequences on the beam.

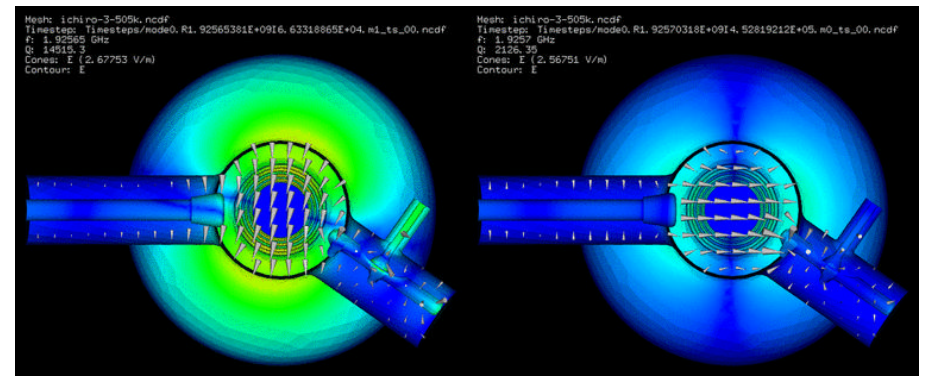

Figure 5 Snapshot of two rotating modes in the ILC LL cavity

\subsection{Parallel meshing}

It is of great interest to model a cryomodule of the ILC that consists of 8-12 SRF cavities. This endeavor will far exceed the single CPU memory limitation of existing meshing software. A parallel meshing capability based on solid model has been developed together with $U$ of Wisconsin and Sandia [10] that makes use of the CHACO graph library to partition the geometry into subdomains and then distribute them onto different processors. Figure 5 demonstrates the test case for a simplified ILC cavity with four sub-domains showing the efficacy of the method.
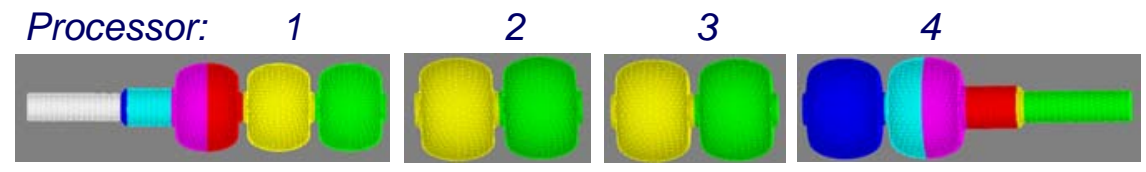

Figure $5 \mathrm{CAD}$ based partitioning of the ILC cavity for paralleling meshing 


\subsection{Shape Optimization}

While simulation is playing an essential role in accelerating cavity design, shape optimization is still a manual, iterative process. A SciDAC collaboration involving SLAC, CMU, Columbia, LBNL, SNL and LLNL is developing a parallel, automated shape optimization capability [11] in Omega3P by bringing together DOE's expertise in large-scale PDE-constrained optimization, in adaptive mesh control and mesh smoothing, and in cavity design. An ongoing application concerns the trapped dipole mode in the ILC LL cavity design as shown in Figure 6 (Left). The goal is to optimize the end cells of the cavity so that there is more mode energy there to increase the damping by the HOM couplers while at the same time not changing the properties of the accelerating mode. Preliminary result from a primitive prototype of this new capability is shown in figure 6 (Right) and further work is in progress.
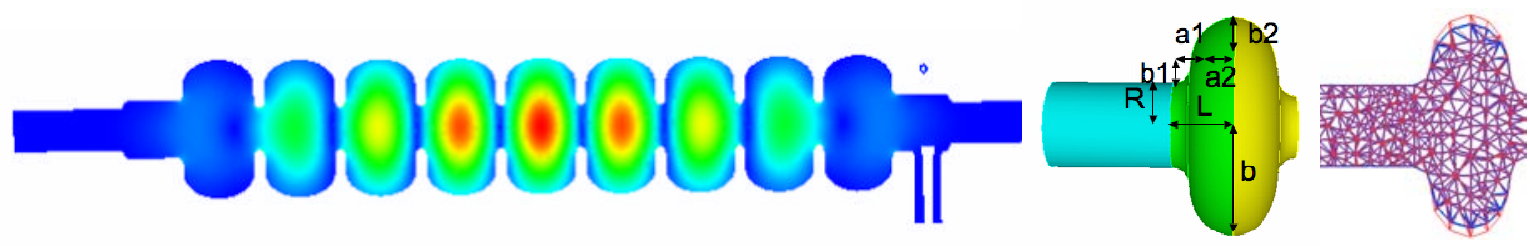

Figure 6 (Left) The field pattern of a well-trapped mode in the ILC Loss-Loss cavity showing little fields in the end cells, (Right) design variables of the end cell and the optimized geometry.

\section{Summary}

The combination of FE method and high performance computing coupled with achievements in complex solvers, visualization, parallel meshing, and shape optimization have led to advances in electromagnetic modeling that can enable simulations of accuracy, problem size, and solution speed previously not possible. This new capability made possible by the SciDAC AST project is presently the core cavity design tool for the ILC R\&D.

\section{References}

[1] http://www.scidac.org/.

[2] K. Ko et el., Impact of SciDAC on Accelerator Projects across the Office of Science through Electromagnetic Modelling, Proc. of SciDAC 2005 Conference, San Francisco, 2005.

[3] J. Sekutowicz et.al., Design of a Low Loss SRF Cavity for the ILC, Proceedings of Particle Accelerator Conference 2005, Knoxville, Tenn., May 15-20, 2005.

[4] http://www.interations.org/linearcollider/.

[5] KEK internal report.

[6] TESLA Technical Design Report, http://tesla.desy.de/new_pages/TDR_CD/.

[7] Z. Bai and Y. Su, SOAR, A Second-order Arnoldi Method for the Solution of the Qaudratic Eigenvalue Problem, SIAM Journal on Matrix Analysis and Applications, Vol. 26, No. 3, pp 640-659, 2005.

[8] L. Lee, et. al., Modeling RF Cavities with External Coupling SIAM Conference on Computational Science and Engineering, Orlando, Florida

[9] K. Ma et al, Scientific Discovery through Advanced Visualization, Proc. of SciDAC 2005 Conference, San Francisco 2005.

[10] T. Tautges et.al., Geometry and Mesh Components for Parallel Applications, Proc. of SciDAC 2005 Conference, San Francisco, 2005.

[11] V. Alcelik et.al., Adjoint Methods for Electromagnetic Shape Optimization of the Low-Loss Cavity for the International Linear Collider, Proc. of SciDAC 2005 Conference, San Francisco 2005 . 